\title{
Inhaltsverzeichnis.
}

Vorwort . . . . . . . . . . . . . . . III

Inhaltsverzeichnis .............. $\mathrm{V}$

Aus der Medizinischen Universitätsklinik (Direktor:

Geh. Med.-Rat Prof. Dr. Fr. Schultze).

Krankheiten der Lungen, der Bronchien und der Pleura.

Von Geh. Med.-Rat Prof. Dr. Fr. Schultze . . . . . I-I

Lungenent $z$ ündungen

Die krupöse Form I - Lobuläre Pneumonie, Bronchopneumonie 3 - Lungenbrand 4 - Lungentuberkulose 4 - Lungenerweiterung, Lungenemphysem 9 Lungenōdem 10.

Erkrankungen der Bronchien

Bronchitis chronica II - Bronchialasthma 12.

Krankheiten der Pleura

Trockene Pleuritis 14 - Exsudative Pleuritis I5 Empyem i6 - Pneumothorax und Pyopneumothorax 17.

HerzgefäBerkrankungen. Von Prof. Dr. Rud. Finkelnburg I8-29 Chronische organische Herzerkrankungen $18-$ Allgemeine Maßregeln bei Herzmuskelinsuffizienz 18 Besondere Indikationen bei einzelnen Herz- und Gefäßkrankheiten 23 -- Arteriosklerose 23 -- Fettherz 24 Akute Erkrankungen des Herzens 25 - Herzgefäßneurosen 28.

Erkrankungen des Mundes und der Speiseröhre. Von Prof.

Dr. Rud. Finkelngurg . . . . . . . . . . . . . . Krankheiten der Mundhöhle 29 - der Speiseröhre 30.

Magenerkrankungen. Von Prof. Dr. Rud. Finkelnburg Akuter Magenkatarrh (Gastritis acuta) 3 I - Chronischer Magenkatarrh (Gastritis chronica) 32 - Ulcus ventriculi (rundes Magengeschwür) 34 - Anomalien der Sekretion des Magensaftes 36 - Nervöse Dyspepsie 39 - Motilitätsstörungen des Magens 40 Gastroptose (Enteroptose) 42 - Magenkarzinom 42.

Darmerkrankungen. Von Prof. Dr. Rud. Finkelnburg . 42-53 Akuter Darmkatarrh (Enteritis acuta) 42 - Chronischer Darmkatarrh (Enteritis chronica) 43 - En- 
teritis membranacea 45 - Chronische (habituelle) Obstipation 46 - Die akute und chronische Appendizitis 48 - Geschwürige Prozesse des Darmes 49 - Neubildungen des Darmes 49 - Nervöse Darmstörungen 49 - Enteroptose 49 - Verengerungen und Verschließungen des Darmes 50 - Hãmorrhoiden 51 - Die tierischen Parasiten des Darmes 51.

Erkrankungen des Peritoneums. Von Prof. Dr. Rud. FINKELNBURG

Akute Peritonitis 53 - Chronische Peritonitis $54-$ Aszites (Bauchwassersucht) 55 .

Erkrankungen der Leber, Gallengänge und Gallenblase und der Pfortader. Von Geh. Med.-Rat Prof. Dr. Fr. Schultze

Erkrankungen der Leber 55 - Erkrankungen der Gallenwege und der Gallenblase 59 - Krankheiten der Pfortader 62.

Pankreaserkrankungen. Von Prof. Dr. Rud. FinkelnBURG

Seite

$53-55$

$55-62$

Erksankungen des Blutes und der blutbereitenden Organe. Von Prof. Dr. H. Stursberg. . . . . . . . . . . .

Polycythaemia vera 64 - Die Anämien $64-$ Die Leukämien 67 - Pseudoleukämische Zustände 68 Die hämorrhagischen Diathesen 68 - Purpura $69-$ Hämophilie 70 .

Erkrankungen des Stoffwechsels. Von Prof. Dr. H. StursBerG . . . . . . . . . . . . . . . . . 70-80

Fettsucht $70-$ Diabetes mellitus $71-$ Gicht $77-$ Diabetes insipidus 79.

Krankheiten der Bewegungsorgane. Von Prof. Dr. Rud. FinKelNBURG . . . . . . . . . . . . . . . . . .

Akuter Gelenkrheumatismus (Polyarthritis rheumatica acuta) 81 - Spezifische (postinfektiöse) Arthritiden 82 - Chronischer Gelenkrheumatismus und Arthritis deformans 82 - Akuter und chronischer Muskelrheumatismus $84-$ Osteomalazie 84 .

Behandlung der organischen Nervenkrankheiten. Von Geh. Med.-Rat Prof. Dr. Fr. Schultze . . . . . . . . . 85-io)

Gehirnkrankheiten 85 - Erkrankungen der Rückenmarkshäute und des Rückenmarks 90 - Krankheiten des peripheren Nervensystems 99.

Neurosen (auber Neurasthenie, Hysterie und Epilepsie). Von Prof. Dr. Rud. Finkelnburg . . . . . . . . . IOl-II4 Hemikranie (Migräne) $101-$ Kopfschmerz (Cephalea) 102 - Angioneurosen (und Trophoneurosen) 
IO3 - Raynaudsche Krankheit (symmetrische GanSeite grän) 103 - Erythromelalgie 104 - Parkinsonsche Krankheit (Paralysis agitans) 104 - Ortliche Muskelkrämpfe 104 - Chorea minor (Veitstanz) I07 - Chorea hereditaria (Huntingtonsche Krankheit) 108 Neuralgien 108 - Basedowsche Krankheit 111 Myxödem II 3 - Akromegalie II 3 - Hemiatrophia facialis 114 .

Krankheiten des Säuglingsalters. Von Prof. Dr. J. EsSer 114-132 Ernährung des Säuglings 114 - Krankheiten des Neugeborenen 118 - Krankheiten des Sāuglingsalters 121 .

Aus der Medizinischen Universitätspoliklinik (Direk-

$$
\text { tor: Prof. Dr. Paul Krause). }
$$

Behandlung der Infektionskrankheiten. Von Prof. Dr. PAUL

KRAuse . . . . . . . . . . . . . . . . . . . . I33-164

Prophylaxe und allgemeine Behandlung von Infektionskrankheiten

Krankenpflege 134 - Diät I 35 - Heilsera und Injektionstechnik I 36 - Antipyretische Behandlung 137 - Verfahren bei infizierten Leichen 137.

Spezielle Behandlung von Infektionskrankheiten

Masern 138 - Scharlach, Röteln I 39 - Windpocken, Pocken 140 - Erysipel 14 I - Septische Erkrankungen 142 - Abdominaltyphus I $44-$ Paratyphus $146-$ Flecktyphus I 46 - Cholera asiatica 146 - Cholera nostras I 48 - Amöbenruhr I 48 - Bazillenruhr 149 Influenza I 50 - Keuchhusten I 5 I - Parotitis epidemica 152 - Angina acuta 153 - Diphtherie 153 - Genickstarre 155 - Kinderlähmung 156-Tetanus I 56 - Aktinomykose 158 - Milzbrand 158 - Maulund Klauenseuche I 58 - Rotz I 59 - SchweiBfriesel 159 - Lyssa 159 - Botulismus 160 - Beri-Beri 160 - Lepra 161 - Malaria 161 - Schwarzwasserfieber 163 - Schlafkrankheit $16_{3}$-- Febris recurrens 163 - Pest 164.

Nierenkrankheiten. Von Prof. Dr. Paul hrause . . . . 164-175 Allgemeine Behandlung der entzündlichen Nierenerkrankungen 164 - Behandlung der verminderten Wasserausscheidung I65 - Akute Nephritis $169-$ Behandlung der chronischen Nephritis inkl. der Schrumpfniere 170 - Stauungsniere 171 - Nierentuberkulose 171 - Nephrolithiasis 172 - Nervöse Nierenkrankheiten 173 - Renale Hämophilie 173 Wanderniere 173 - Pyelitis 174 - Erkrankungen der Nebennieren 174. 
Erkrankungen der Harnblase und der funktionellen Stō- Seite rungen der männlichen Geschlechtsorgane. Von Prof.

Dr. Paul Krause. . . . . . . . . . . . 175-180

Erkrankung der Harnblase I75 - Funktionelle Erkrankungen der männlichen Harn- und Geschlechtsorgane 179 .

Aus der Medizinischen Klinik (Direktor: Geh. Med.-

Rat Prof. Dr. Fr. Schultze) und der Klinik für

Psychisch- und Nervenkranke (Direktor: Geh.

Med.-Rat Prof. Dr. A. WestPhal).

Therapie der Neurosen: Neurasthenie, Hysterie, Epilepsie. Von Geh. Med.-Rat Prof. Dr. A. Westphal, Prof. Dr.

R. Finkelnburg und Prof. Dr. A. H. Hübner . . . I81-194

Neurasthenie 181 - Traumatische Neurasthenie 186 - Hysterie 186 - Epilepsie I90.

Aus der Psychiatrischen und Nervenklinik (Direktor:

Geh. Med.-Rat Prof. Dr. A. WestPhal).

Psychiatrische Klinik. Von Geh. Med.- Rat Prof. Dr. A.

Westphal und Prof. Di. A. H. Hubner.... . 195-213

Allgemeine Therapie 195.

Spezielle Therapie:

Melancholie 200- Manie 201 - Degeneration 202 -

Hypochondrie 202 - Traumatische Psychosen 202 -

Paranoia chronica 203 - Paranoia acuta 204 -

Querulantenwahn 204 - Induziertes Irresein 204 -

Symptomatische Psychosen 204 - Imbezillität und

Idiotie, Kretinismus, Myxödem 205 - Hebephrenie,

Katatonie, Dementia paranoides 206-Alkoholpsychosen 207 - Delirium tremens 207 - Alcoholismus chronicus 207 - Morphinismus 208 - Kokainismus 209 - Dementia paralytica 210 - Hirnsyphilis 210 Arteriosklerose 212 - Senile Geistesstörungen 2 I 3.

Aus der Chirurgischen Klinik (Direktor: Geh. Med.-

$$
\text { Rat Prof. Dr. GarRè). 214-352 }
$$

Allgemeiner Teil. Von Dr. H. FrUnd . . . . . . . . 214-220

Frische Verletzungen. Von Dr. H. FRüND . . . . . . 220-226

Chirurgische Infektionskrankheiten (mit A usnahme der

Tuberkulose) und Gangrän. Von Dr. H. Geinırz 227-24I 
Furunkel 227 - Abszesse 228 - Erysipel 228 Seite Phlegmone 229 - Osteomyelitis 233 - Chronische Form der Osteomyelitis 235 - Thrombose, Thrombophlebitis, Lymphangitis und -adenitis 235 - Sehnenscheidenentzündungen 236 - Gelenkergüsse 236 Syphilis 237 - Milzbrand 237 - Aktinomykose 238 - Gangrān 238 - Ulcus cruris, Varizen 240.

Tuberkulose. Von Dr. M. KRABBEL . . . . . . . . . . 241-247

Kopf und Hels. Von Dr. R. Syring . . . . . . . . 247-277

Kopfschwarte 248 - Knōcherner Schädel 249 Hirnhäute und Gehirn 250 - Gesicht und Gesichtsşchādel 256 - Weichteile des Gesichts 256 - Nase und Nebenhöhlen 261 - Kiefer und Zähne 262 Mund und Rachen 265.

Weichteile des Halses 270 - Kehlkopf 273 - Schilddrüse 275 .

Erkrankungen der Mamma und der inneren Brustorgane.

Von Dr. H. Els . . . . . . . . . . . . . . . 277-285 Mamma 277 - Osophagus 278 - Trachea und Bronchien 280 - Lungen und Pleura 28 $\mathrm{I}$ - Herzverletzungen 285 .

Erkrankungen des Bauches. Von Dr. H. Els . . . . . 285-302

Bauchverletzungen 285 - Bauchdecken 286 - Brüche 286 - Peritoneum 289 - Appendizitis 29J - Erkrankungen der Leber und des Gallensystems 293 Pankreaserkrankungen 295 - Magenerkrankungen 296 - Darmerkrankungen 298 - Erkrankungen des Mastdarms und des Afters 300 .

Chirurgische Behandlung der Erkrankungen der Harn- und

Geschlechtsorgane. Von Dr. R. BAYER ... . . . 303-319

Erkrankungen der Nierenkapsel 303 - der Niere selbst 305 - des Harnleiters 307 - der Harnblase 308 - der Prostata 313 - der Harnröhre 314 - des Penis und der Vorhaut 316 - des Hodens, Nebenhodens und Samenstranges $317-$ des Skrotums und der Scheidenhāute 319.

Therapie der Frakturen und Lurationen. Von Prof. Dr.

A. Machol . . . . . . . . . . . . . . 320-336

Frakturen der oberen Extremität 320 - der unteren Extremität 326 - Beckenbrüche $330-$ Brüche des Schultergürtels 33I - Rippenbrüche 33I - Brustbeinbrüche $33 \mathrm{I}$ - Schädelbrüche $33 \mathrm{I}$ - Brüche der Gesichtsknochen 331 - Wirbelbrüche 332 - Luxationen der oberen Extremität 332 - der unteren Extremität 334 - der Wirbelsäule 336. 
Orthopädische Therapie. Von Prof. Dr. A. MAchol Angeborene Deformitäten $33^{6}-$ Fußdeformitäten 340 - Erworbene Deformitäten 342 - Deformitäten der Hand und Finger 345 - Deformitäten im Anschluß an Entzündungen 349 - Deformitäten auf neurogener Basis 349 .

Aus der Augenklinik (Direktor: Geh. Med.-Rat Prof. Dr. H. KuHNT).

Therapie der Augenkrankheiten. Von Geh. Med.-Rat Prof.

Dr. H. Kunnr (Mit 7 Abbildungen) . . . . . . . 353-402

Allgemeine Vorbemerkungen 353 -- Erkrankungen der Orbita 356 - der tränenableitenden Wege $357-$ der Lider 359 - der Bindehaut 365 - der Sklera 376 - der Hornhaut 377 - der Gefäß- und der Netzhaut 386 - der Linse 391 - des Glaskörpers 393 - des Nervus opticus 393 - Strabismus $39^{8}$ - Verletzungen 399 - Ophthalmia sympathica 402.

Die Therapie der Universitätspoliklinik für Ohren-,

Hals- und Nasenkranke. Von Geh. Med.-Rat Prof. Dr. H. WALB

Ohrenkrankheiten:

Allgemeiner Teil 403 - Spezieller Teil: Ohrmuschel 404 - Außerer Gehörgang 405 - Trommelfell 407 Mittelohr 408 - Ohrtrompete 4I 3 - Labyrinth 4I4.

Nasenkrankheiten:

Allgemeiner 'Teil 4I 5 - Spezieller Teil: Erkrankungen der Nasenschleimhaut 416 - der Nasenscheidewand $4 \mathrm{I} 7$ - der Nebenhöhlen 4I8.

Rachenkrankheiten:

Allgemeiner Teil 421 - Spezieller Teil : Erkrankungen der Gaumenmandeln 422 - der Rachenmandeln 424 - Tumoren des Rachens 425.

Kehlkopfkrankheiten:

Allgemeiner Teil 426 - Spezieller Teil: Geschwüre des Kehlkopfs 427 - Lähmungen der Kehlkopfmuskeln 428 - Verengerungen des Kehlkopfs 428 Neubildungen im Kehlkopf 429.

Aus der Universitäts-Frauenklinik (Direktor: Geh. Rat Prof. Dr. von Franqué).

Geburtshilfe und Gynäkologie. Von Prof. Dr. K. ReifferSCHEID . . . . . . . . . . . . . . . . . . . . . . 430-468

Geburtshilfe:

Leitung der normalen Geburt 430 - der Neugeburtsperiode $43 \mathrm{I}$ - Wochenbett 43 I - Dammriß 432 - 
Asphyxie des Neugeborenen 432 - Tuberkulose und Schwangerschaft 433 - Herzfehler und Schwangerschaft 433 - Retroflexio uteri gravidi 433 - Myom, Karzinom, Ovarialtumor, Appendizitis und Schwangerschaft 434 - Pyelitis 435 - Schwangerschaftsniere 435 - Eklampsie, Hyperemis gravidarum 436 - Icterus gravis, Fehlgeburt 437 - Perforation des Uterus 438 - Habitueller Abort, Der kūnstliche Abort, Blasenmole 439 - Hydramnion, Placenta praevia 440 - Vorzeitige Lösung der rechtssitzenden Plazenta 44I - Die pathologischen Kindeslagen 441 - Nabelschnurvorfall 443 - Hydrocephalus 443 - Die Leitung der Geburt bei engem Becken 443 Nachblutungen 446 - Puerperalfieber 447 Mastitis 448.

$G$ ynäkologie:

Erkrankungen der Vulva 448 - Neubildungen der Vulva 449 - Erkrankungen der Scheide 450 - Neubildungen der Scheide 45I - Verletzungen der Scheide 451 - Entzündungen des Uterus 452 Lageveränderungen des Uterus 456 - Neubildungen des Uterus 46I - Die entzündlichen Erkrankungen der Adnexe 464 - Perimetritis und Parametritis 466 - Extrauteringravidität 466 - Neubildung der Ovarien 467 - Störungen der Menstruation 467.

Aus der Klinik für Hautkrankheiten (Prof. Dr.

$$
\text { ERICH HofFmanN). }
$$

Die Therapie der Haut- und Geschlechtskrankheiten. Von

Priv.-Doz. Dr. W. Friebors . . . . . . . . . . 469-56

Spezielle Mittel 469 - Lichtbehandlung 477 - Verbände 479 - Hautkrankheiten (in alphabetischer Reihenfolge) $48 \curvearrowright-538-$ Geschlechtskrankheiten $539-563$.

\section{An hang:}

Aus der Medizinischen Universitätsklinik (Direktor:

Geh. Med.-Rat Prof. Dr. Fr. Schultze).

Spezielle therapeutische Technik. Von Prof. Dr. H. StursBerg 564-577

Einspritzungen und Punktionen 564 -- Speiseröhre und Magen 572 - Darminfusion 575 - Allgemeines über Behandlung von Blutungen 576 .

Therapie der (klinisch wichtigsten) Vergiftungen. Von Prof.

Dr. R. Finkelnburg . . . . . . . . . . 577-587 Allgemeine Vorbemerkungen $577-$ Die einzelnen Vergiftungen (alphabetisch geordnet) 579.

Sachregister . . . . . . . . . . . 588 
